\title{
Remdesivir-Ivermectin combination displays synergistic interaction with improved in vitro antiviral activity against SARS-CoV-2
}

Laura Jeffreys $^{1 *}$, Shaun H Pennington ${ }^{1 *}$, Jack Duggan ${ }^{1}$, Alastair Breen ${ }^{1}$, Jessica Jinks ${ }^{1}$, Alison Ardrey $^{1}$, Samantha Donnellan ${ }^{1}$, Edward I Patterson ${ }^{1,2}$, Grant L Hughes ${ }^{1,2}$, David Hong ${ }^{3}$, Paul M O’Neill ${ }^{3}$, Ghaith Aljayyoussi ${ }^{1}$, Andrew Owen ${ }^{4}$, Stephen A Ward ${ }^{1}$ and Giancarlo A Biagini $^{1 * *}$

${ }^{1}$ Centre for Drugs and Diagnostics, Department of Tropical Disease Biology, Liverpool School of Tropical Medicine, Liverpool, L3 5QA, UK

${ }^{2}$ Department of Vector Biology, Liverpool School of Tropical Medicine, Liverpool, L3 5QA, $U K$

${ }^{3}$ Department of Chemistry, University of Liverpool, Liverpool, L69 7ZD, U.K.

${ }^{4}$ Department of Pharmacology and Therapeutics, Centre of Excellence in Long-acting Therapeutics (CELT), University of Liverpool, Liverpool, L69 3GE, UK.

\section{*Joint first authors \\ *** Corresponding author}

Giancarlo A Biagini, Centre for Drugs and Diagnostics, Department of Tropical Disease Biology, Liverpool School of Tropical Medicine, UK. Tel: 0151705 3151; email: giancarlo.biagini@1stmed.ac.uk

\footnotetext{
Abstract

A key element to the prevention and management of the COVID-19 pandemic is the development of effective therapeutics. Drug combination strategies of repurposed drugs offer a number of advantages to monotherapies including the potential to achieve greater efficacy, the potential to increase the therapeutic index of drugs and the potential to reduce the emergence of drug resistance. Combination of agents with antiviral mechanisms of action with immune-modulatory or anti-inflammatory drug is also worthy of investigation. Here, we report on the in vitro synergistic interaction between two FDA approved drugs, remdesivir (RDV) and ivermectin (IVM) resulting in enhanced antiviral activity against SARS-CoV-2, the causative pathogen of COVID-19. These findings warrant further investigations into the clinical potential of this combination, together with studies to define the underlying mechanism.
} 


\section{Introduction}

As of the end of 2020, the WHO has reported more than 76 million confirmed cases of COVID19 , including over 1.6 million deaths ${ }^{1}$. As part of an overarching strategy that includes the development of effective vaccines and rapid diagnostics, considerable efforts are underway to identify therapeutics to prevent and treat COVID-19. Potential therapeutic strategies may include investigating the repurposing of existing drugs to the discovery of novel therapies. Thousands of clinical trials are currently underway, with therapeutic approaches involving direct-acting antivirals, for the prevention of virus replication, and host-directed therapies aimed at mitigating against the pathology of the disease ${ }^{2,3}$.

Towards these efforts, strategies to identify effective combination therapies are emerging, with several laboratories reporting in vitro combination screens ${ }^{4}$ and in vivo animal combinations studies ${ }^{5}$. A large number of clinical trials are also evaluating combination approaches ${ }^{6}$. There are several advantages of effective drug combinations, including: (i) achieving greater efficacy (reducing mortality and morbidity), (ii) prevention/minimisation of the emergence of drug resistance, and (iii) increasing the therapeutic index of drugs that show synergistic antiviral effects. Importantly, even in the absence of synergy an additive antiviral interaction for two drugs with separate mechanisms of action may profoundly reduce the speed at which drug resistance is transmitted. An example of the benefits of a drug combination approach can be seen in HIV treatment, where antiretroviral combination drug treatment has resulted in dramatic improvements in both patient morbidity and mortality ${ }^{7}$. Other clear examples also exist for other viruses, where HCV became the first chronic viral disease to become curable with drug combinations.

Two repurposed drugs that have received notable attention for the treatment of COVID-19 include remdesivir (RDV) and ivermectin (IVM). $\mathrm{RDV}$ is a prodrug $\mathrm{C}$-adenosine nucleoside analog that inhibits the viral RNA-dependent, RNA polymerase which has demonstrated in vitro inhibitory activity against SARS-CoV-1 and Middle East respiratory syndrome (MERS$\mathrm{CoV})^{8,9}$. RDV was shown early in the pandemic to display in vitro antiviral efficacy against SARS-CoV-2 ${ }^{10}$ and has subsequently been investigated in several human clinical trials. In a recently published double-blind, randomized, placebo-controlled trial, intravenous administration of RDV showed superiority relative to placebo in shortening the time to recovery in adults who were hospitalized with COVID-19 ${ }^{11}$. RDV is currently the only FDA licenced drug approved for the treatment of COVID-19, but on $20^{\text {th }}$ November 2020 the WHO issued a conditional recommendation against the use of RDV in hospitalised patients (irrespective of disease severity) because there is no evidence supporting an improvement in survival or other outcomes in these patients. A combination of antivirals, and/or a combination between antivirals and anti-inflammatory/immunomodulatory agents may have more success.

IVM is an FDA-approved anti-parasitic drug active against a wide range of parasites, including gastrointestinal roundworms, lungworms, mites, lice, hornflies and ticks ${ }^{12}$. In addition, IVM is reported to exhibit broad spectrum anti-viral activity against a wide range of RNA and DNA viruses ${ }^{13}$. Recently, IVM was also shown to display anti-viral activity against SARS-CoV-2 ${ }^{14}$, but approved doses are not expected to be high enough to achieve in vitro-defined target exposures systemically ${ }^{15}$. Several clinical trials are now evaluating the potential of IVM for both prophylaxis and treatment of COVID-19, but the low exposures make the antiinflammatory and/or immunomodulatory mechanisms of action more plausible than a direct antiviral activity of the monotherapy ${ }^{16}$. In nematodes and insects, IVM has been shown to target glutamate-gated $\mathrm{Cl}^{-}$channels $\left(\mathrm{GUCl}^{-},{ }^{13}\right)$, whilst its antiviral properties may be the result of interaction with viral and/or host factors. For example, IVM displays anti-flavivirus activity 
via inhibition of the viral NS3 helicase ${ }^{17}$. In addition, IVM has also been shown to inhibit classical nuclear transport of viral proteins (e.g. DENV NS5) via disruption of the importin $\alpha / \beta$ heterodimer ${ }^{14}$. However, studies with SARS-CoV-2 in Syrian Golden Hamsters showed an impact upon disease pathology in the absence of any effect on viral titres ${ }^{18}$.

Here, using a classic combination testing approach to determine the Fractional Inhibitory Concentration Index (FICI), we report a synergistic interaction between RDV and IVM resulting in improved in vitro antiviral activity against SARS-CoV-2. The data are discussed in the context of current therapeutic efforts against COVID-19.

\section{Methods}

SARS-CoV-2 Strain. SARS-CoV-2/Human/Liverpool/REMRQ0001/2020 was isolated from a nasopharyngeal swab from a patient in Liverpool and passaged a further 4 times in Vero E6 cells. The mapped RNA sequence has previously been submitted to Genbank, accession number MW041156.

VERO E6 cell culture and plate preparation. VERO E6 cells were maintained in complete EMEM (EMEM supplemented with 10\% heat-inactivated FBS [Gibco; 10500-064] and 1\% penicillin/streptomycin [Gibco; 15140-122]) at $37{ }^{\circ} \mathrm{C}$ with $5 \% \mathrm{CO}_{2}$. VERO E6 cells were not allowed to exceed $80 \%$ confluence in culture and were not propagated beyond passage 15 . Cells were harvested from flasks by removing the media, washing with PBS, and incubated them at $37{ }^{\circ} \mathrm{C}$ with $5 \% \mathrm{CO}_{2}$ for 10 min with trypsin-EDTA (Sigma-Aldrich; T4049). Cells were transferred to $50 \mathrm{~mL}$ centrifuge tubes in resting EMEM (EMEM supplemented with 10 $\%$ heat-inactivated FBS) and enumerated. Cells were seeded in resting EMEM at $1 \times 10^{5}$ cells/well in 96-well plates (Grenier Bio-one; 655090). Plates were then incubated for $20 \mathrm{~h}$ at $37{ }^{\circ} \mathrm{C}$ with $5 \% \mathrm{CO}_{2}$ to allow the cells to reach $100 \%$ confluence. The resting minimal medium was then removed, and the cells used for downstream applications.

Measurement of drug efficacy against SARS-Cov-2 and assessment of the Fractional Inhibitory Concentration Index for RDV-IMC combinations. In order to generate EC50s for RDV and IVM, VERO E6 cells were treated with either drug in minimal medium at 25.00 $\mu \mathrm{M}, 8.33 \mu \mathrm{M}, 2.78 \mu \mathrm{M}, 0.93 \mu \mathrm{M}, 0.31 \mu \mathrm{M}, 0.10 \mu \mathrm{M}$ and $0.03 \mu \mathrm{M}$ (DMSO maintained at $0.25 \%$ ) or control media, as appropriate in 96 -well plates. The plates were then incubated at $37^{\circ} \mathrm{C}$ with $5 \% \mathrm{CO}_{2}$ for $2 \mathrm{~h}$ to mimic prophylaxis treatment. Following this incubation, the minimal medium containing the experimental compounds and the control media was removed and wells were then treated with $50 \mu \mathrm{L}$ minimal medium containing SARS-CoV-2 (MOI of 0.05 ). After addition of virus, $100 \mu \mathrm{L} 2 \times$ semi-solid media and then $50 \mu \mathrm{L}$ minimal medium containing experimental compounds and control media, as appropriate were added. The plates were allowed to incubate for $48 \mathrm{~h}$, and then fixed by the addition of $4 \%$ paraformaldehyde for $1 \mathrm{~h}$ at room temperature. Following fixation, the media from each well was removed and cells stained with $0.25 \%(\mathrm{w} / \mathrm{v})$ crystal violet. Following $(3 \mathrm{x})$ washing with $\mathrm{dH}_{2} \mathrm{O}_{2}$, cytopathic viral activity was determined by measuring absorbance of each well at $590 \mathrm{~nm}$ using a Varioskan LUX microplate reader (Thermo Fisher Scientific).

Following the assessment of the inhibitory effect $\left(\mathrm{EC}_{50}\right)$ of RDV and IVM monotherapy on the cytopathic viral activity of SARS-CoV-2, combination studies and analyses of the Fractional Inhibitory Concentration Index (FICI) were undertaken using the method of Berenbaum ${ }^{19}$. Interpretation of FICI $(\mathrm{FICI}<=0.5=$ synergy; FICI $>4.0=$ antagonism; FICI $>0.5-4=$ no interaction) follows guidance provided by the Journal of Antimicrobial Chemotherapy ${ }^{20}$. All 
monotherapy and combination drug sensitivity experiments were performed using three technical replicates and using at least two independent biological replicates.

\section{Results}

Initial experiments were conducted to confirm the anti-SARS-CoV-2 activity of IVM and RDV monotherapies. Dose-effect relationship for the inhibition (\%) of SARS-CoV-2 cytopathic activity for both drugs is shown in Fig. 1. The measured $\mathrm{EC}_{50}$ for IVM was $2.3 \pm 1.0 \mu \mathrm{M}$ and EC50 for RDV was $1.9 \pm 1.4 \mu \mathrm{M}$. These values closely match those reported in recent studies for both drugs ${ }^{10,14}$.

Subsequent determination of the IVM-RDV combination interaction using isobologram analysis revealed a $\mathrm{FICI}<=0.5$ indicating a synergistic interaction (Fig. 2).

\section{Discussion}

Here, we described the synergistic interaction between two FDA approved drugs resulting in enhanced in vitro antiviral activity against SARS-CoV-2, the causative pathogen of COVID19. Although combination therapy offers a number of explicit advantages, genuine descriptions of synergy are relatively infrequent ${ }^{21}$. This observation appears to also be relevant to SARS-CoV-2 as there have been very few reports of validated synergistic interactions, despite thousands of combination experiments e.g. ${ }^{4,22}$.

At this stage, the mechanism underpinning the synergistic interaction between RDV and IVM resulting in improved in vitro efficacy against SARS-CoV-2 is unclear. Both drugs have previously been shown to inhibit SARS-CoV-2 replication ${ }^{10,14}$. Given that RDV has a welldefined mechanism of action and is known to inhibit the RNA-dependent, RNA polymerase ${ }^{8}$, it will be of interest to investigate whether IVM provides synergy by also inhibiting an as yet defined alternative but complimentary function of RNA replication in SARS-CoV-2.

At the doses currently being investigated for SARS-CoV-2, the known plasma pharmacokinetics suggest that concentrations sufficient to inhibit viral replication will not be achieved in plasma ${ }^{15}$. However, modelling approaches indicate that inhibitory concentrations may be achieved in lung ${ }^{15}$, although this has been questioned by other authors ${ }^{23}$. Nonetheless, a combination containing IVM may offer the opportunity to exploit the anti-inflammatory and/or immunomodulatory activity of this agent, while simultaneously augmenting the antiviral activity of viral RNA polymerase inhibitors like RDV. If this synergy is also evident with orally bioavailable polymerase inhibitors such as favipiravir or molnupiravir, the approach may be more widely exploitable for community deployment in earlier disease. However, given the low exposures of IVM, an additional drug with an achievable antiviral mechanism of action may also help stem emergence and transmission of resistance.

Further investigation is now required to determine whether the observed synergistic interaction can be replicated in animal models of COVID-19 disease, and if these results are favourable, whether the finding can be translated to the clinic. It remains to be determined whether or not the synergistic RDV-IVM interaction will result in a favourable shift in the plasma $\mathrm{C}_{\max } / \mathrm{EC}_{90}$ ratio. Nevertheless, the underpinning mechanisms for this synergy warrant further investigation so that this pharmacodynamic advantage can be exploited for future development of optimal drug combinations.

Acknowledgements. GAB and SAW acknowledge support from the Medical Research Council (MR/ 836 S00467X/1). GAB, SAW and GLH acknowledge support from the UK 
Research and Innovation (UKRI) Strength in Places Fund (SIPF 20197). A.O. acknowledges research funding from Unitaid (LONGEVITY) and EPSRC (EP/R024804/1).

\section{References}

1. WHO. WHO Coronovirus Disease (COVID-19) Dashboard. https://covid19whoint/ 2020.

2. McKee DL, Sternberg A, Stange $U$ et al. Candidate drugs against SARS-CoV-2 and COVID-19. Pharmacol Res 2020; 157: 104859.

3. Sanders JM, Monogue ML, Jodlowski TZ et al. Pharmacologic Treatments for Coronavirus Disease 2019 (COVID-19): A Review. JAMA : the journal of the American Medical Association 2020; 323: 1824-36.

4. Ianevski A, Yao R, Biza S et al. Identification and Tracking of Antiviral Drug Combinations. Viruses 2020; 12.

5. Kaptein SJF, Jacobs $S$, Langendries $L$ et al. Favipiravir at high doses has potent antiviral activity in SARS-CoV-2-infected hamsters, whereas hydroxychloroquine lacks activity. Proc Natl Acad Sci U S A 2020; 117: 26955-65.

6. Kalil AC, Patterson TF, Mehta AK et al. Baricitinib plus Remdesivir for Hospitalized Adults with Covid-19. N Engl J Med 2020.

7. Palella FJ, Jr., Delaney KM, Moorman AC et al. Declining morbidity and mortality among patients with advanced human immunodeficiency virus infection. HIV Outpatient Study Investigators. N Engl J Med 1998; 338: 853-60.

8. Agostini ML, Andres EL, Sims AC et al. Coronavirus Susceptibility to the Antiviral Remdesivir (GS-5734) Is Mediated by the Viral Polymerase and the Proofreading Exoribonuclease. mBio 2018; 9.

9. Sheahan TP, Sims AC, Graham RL et al. Broad-spectrum antiviral GS-5734 inhibits both epidemic and zoonotic coronaviruses. Science translational medicine 2017; 9.

10. Pizzorno A, Padey B, Dubois J et al. In vitro evaluation of antiviral activity of single and combined repurposable drugs against SARS-CoV-2. Antiviral research 2020; 181: 104878.

11. Beigel JH, Tomashek KM, Dodd LE et al. Remdesivir for the Treatment of Covid-19 Final Report. N Engl J Med 2020; 383: 1813-26.

12. Crump A, Omura S. Ivermectin, 'wonder drug' from Japan: the human use perspective. Proc Jpn Acad Ser B Phys Biol Sci 2011; 87: 13-28.

13. Heidary F, Gharebaghi R. Ivermectin: a systematic review from antiviral effects to COVID-19 complementary regimen. J Antibiot (Tokyo) 2020; 73: 593-602.

14. Caly L, Druce JD, Catton MG et al. The FDA-approved drug ivermectin inhibits the replication of SARS-CoV-2 in vitro. Antiviral research 2020; 178: 104787.

15. Arshad U, Pertinez $\mathrm{H}$, Box $\mathrm{H}$ et al. Prioritization of Anti-SARS-Cov-2 Drug Repurposing Opportunities Based on Plasma and Target Site Concentrations Derived from their Established Human Pharmacokinetics. Clinical pharmacology and therapeutics 2020; 108: 775-90.

16. Steinhoff $M$, Vocanson $M$, Voegel JJ et al. Topical Ivermectin $10 \mathrm{mg} / \mathrm{g}$ and Oral Doxycycline $40 \mathrm{mg}$ Modified-Release: Current Evidence on the Complementary Use of AntiInflammatory Rosacea Treatments. Adv Ther 2016; 33: 1481-501.

17. Mastrangelo $E$, Pezzullo $M$, De Burghgraeve T et al. Ivermectin is a potent inhibitor of flavivirus replication specifically targeting NS3 helicase activity: new prospects for an old drug. J Antimicrob Chemother 2012; 67: 1884-94. 
18. de Melo GD, Lazarini F, Larrous F et al. Anti-COVID-19 efficacy of ivermectin in the golden hamster. bioRxiv 2020: 2020.11.21.392639.

19. Berenbaum MC. A method for testing for synergy with any number of agents. The Journal of infectious diseases 1978; 137: 122-30.

20. Odds FC. Synergy, antagonism, and what the chequerboard puts between them. $J$ Antimicrob Chemother 2003; 52: 1.

21. Greco WR, Bravo G, Parsons JC. The search for synergy: a critical review from a response surface perspective. Pharmacol Rev 1995; 47: 331-85.

22. Riva L, Yuan S, Yin X et al. Discovery of SARS-CoV-2 antiviral drugs through large-scale compound repurposing. Nature 2020; 586: 113-9.

23. Schmith VD, Zhou JJ, Lohmer LRL. The Approved Dose of Ivermectin Alone is not the Ideal Dose for the Treatment of COVID-19. Clinical pharmacology and therapeutics 2020;

108: $762-5$ 
A

B

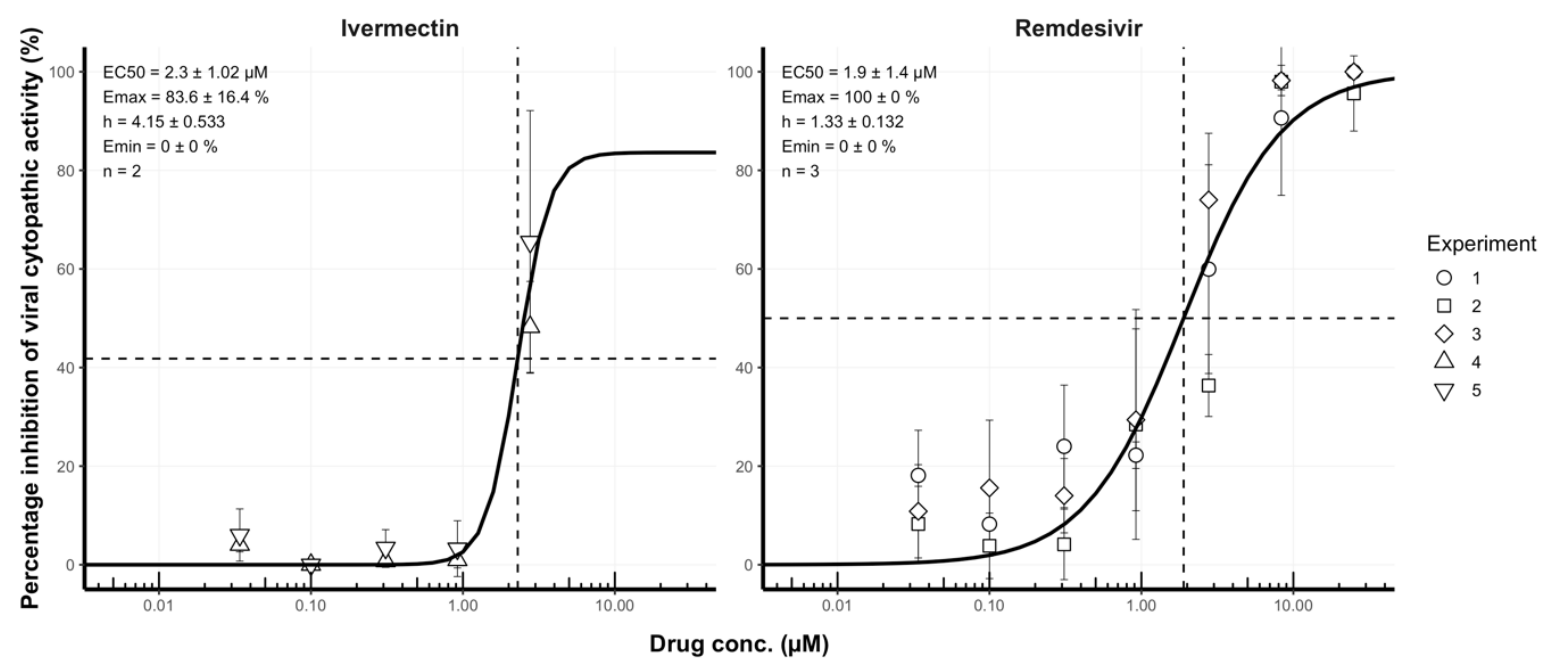

Figure 1. Dose-effect relationship for the inhibition (\%) of SARS-CoV-2 cytopathic activity by (A) Ivermectin (IVM) and (B) Remdesivir (RDV). Non-linear regression was performed on data (mean \pm S.D.) taken from a minimum of two independent biological replicates each performed in triplicate.

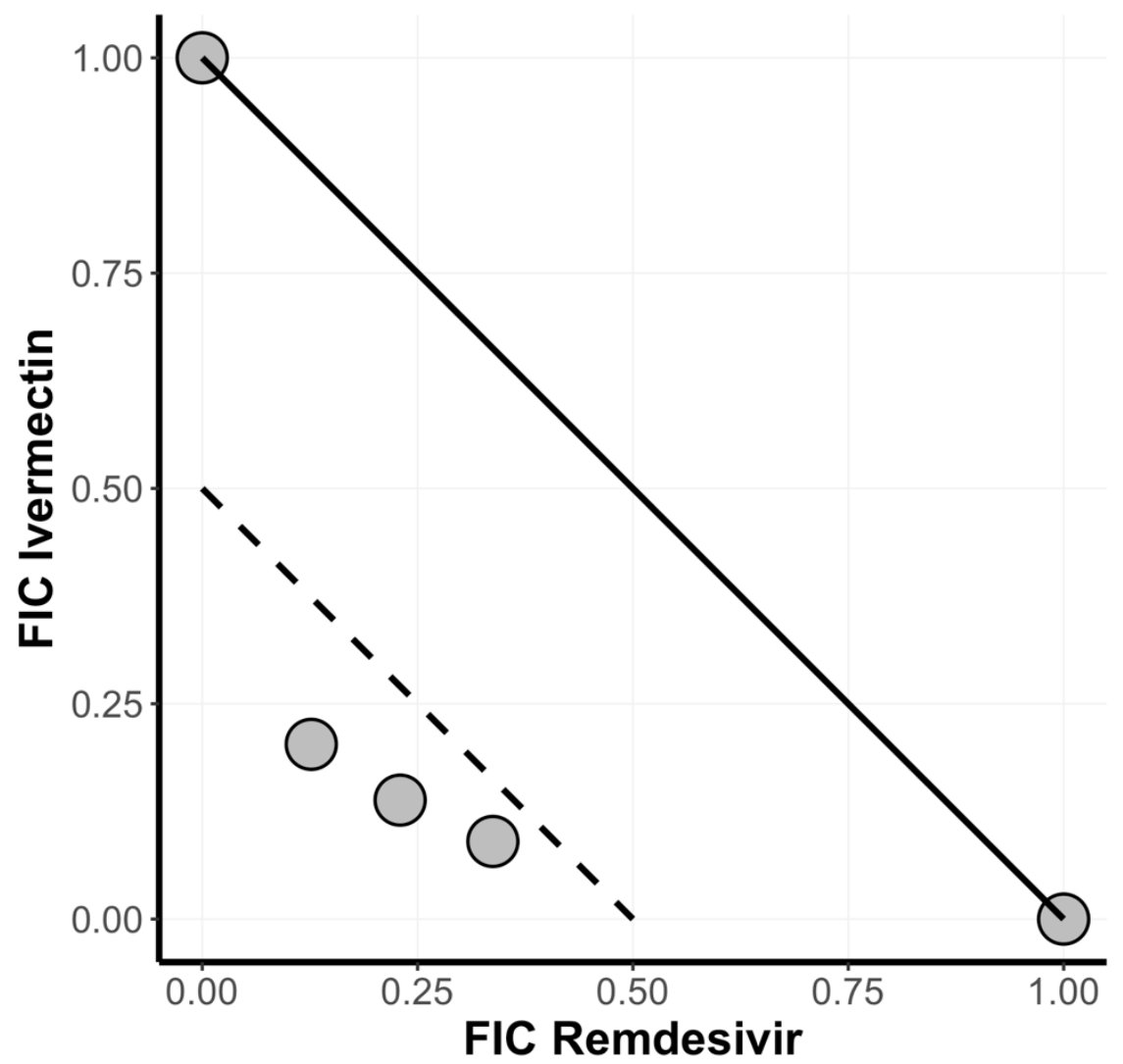

Figure 2. Isobologram showing interactions between Remdesivir and Ivermectin. 